\title{
Study of Some Basic Transport Coefficients in Noble-Gas Discharge Plasmas
}

\author{
M. H. Elghazaly, S. Solyman and A. M. Abdel baky \\ Department of Physics, Faculty of Science, University of Zagazig, \\ Zagazig, Egypt
}

The transport coefficients, electrical and thermal conductivities, have been calculated in argon and helium glow discharge plasmas. The electron collision frequency needs to by know [In order to calculate the transport coefficients]. The three collision mechanisms, electron-neutral, electron-ion and electron-electron collision frequencies, are all investigated. The most important internal parameters of the plasma which have a direct bearing on the calculation of the collision frequency are the electron temperature and density, and neutral atom density. The electron temperature and electron density were determined from measurements taken with a double Langmuir probe. The double probe was placed into the different regions of the glow discharge from the cathode surface to the anode. The measured electron temperature are about $(0.98-6.30 \mathrm{eV})$ at discharge voltage of 400 volt for two different argon pressures 13.3 and $26.6 \mathrm{~Pa}$, and (2.86-8.14 eV) for helium discharge at 750 volt and 53.2 and $79.8 \mathrm{~Pa}$, respectively. The electron density, over the pressure and voltage ranges employed above, are about $\left(1.19-7.65 \times 10^{9} \mathrm{~cm}^{-3}\right)$ and $(6.70-$ $\left.8.18 \times 10^{9} \mathrm{~cm}^{-3}\right)$ for argon and helium plasmas, respectively. In addition, the neutral atom density, $n_{n}$, is computed from the gas pressure and temperature using ideal gas law. Calculated results of electrical and thermal conductivities and their ratios, Wiedemann-Franz ratios, are represented graphically as functions of electron temperature, with the gas pressure as parameter. It was found that electron energy greatly affects the plasma transport coefficients.

\section{1- Introduction:}

The fundamental properties of plasma are markedly dependent upon the interactions of the plasma particles with the force fields existing inside it. Our interest is in weakly ionized plasmas, since a glow-discharge is a selfsustaining, weakly ionized plasma that emits light (i.e., it glows). In these plasmas the electrons tend to dominate the situation, since they respond quickly to the influence of electric and magnetic fields, in view of their low inertia [1]. 
Low-pressure, weakly ionized noble-gas glow discharge plasmas are very frequently subject of both basic and applied research and widely used in industrial technological processes. Low-pressure $(0.13 \mathrm{~Pa}$ to $1333 \mathrm{~Pa})$, cold (gas temperature 300 to $500 \mathrm{~K}$ ), weakly ionized (degree of ionization $10^{-6}$ to $10^{-}$ ${ }^{1}$ and the charge-neutral interactions dominate over the multiple coulomb interactions) glow discharge plasmas are used extensively in the processing of electronic materials, especially for etching and deposition of thin films. Also, application was found in surface modification, e.g., hardening and corrosion resistance [2].

Processes related to the transport of mass, momentum, energy and charges in plasma are generally called transport phenomena. There exist general equations describing these different phenomena, and the special effects are characterized by coefficients generally called transport coefficients [3]. These transport coefficients can be evaluated when the momentum transfer collision frequency is known.

Different types of collision processes have a dominant influence on the transport properties of the plasma. For the electrons, a variety of different collision mechanisms is of importance. Elastic collisions are responsible for momentum loss and limit the electric as well as the heat conductivity. The most dominant mechanism, causing a loss of electron momentum, is elastic electronneutral collisions [4].

Transport phenomena in plasmas can be promoted by external and internal forces. In spatially homogeneous plasma under the influence of external forces, a drifting of the electrons can occur. This motion induced by external forces is referred to as mobility. Since the electrons have electric charge, their motion implies in conduction of electricity when acted upon by an external electric field. On the other hand, the electrons also have kinetic energy associated with their random thermal motion, their drift implies in the transport of thermal energy and therefore in heat conduction [1].

The goal of this work is the calculation of the basic transport coefficients (electrical and thermal conductivities) for argon and helium plasmas. To deduce the transport coefficients, we need only know the momentum transfer collision frequency of the plasma species. Electron-neutral collisions, electron-ion collisions and electron-electron collisions are all investigated. Electron temperature and density, and neutral atom density are all we need to know for the evaluation of the electron collision frequencies. Therefore, we present in our article the measurements of the electron 
temperature and electron density by means of the Langmuir probe technique, in addition to thermal couple for neutral gas temperature.

\section{Transport Coefficients}

Study of the conductivity of the plasma can be achieved by solving the equation of motion for each plasma species. Because electrons have far lower masses than ions, they have far higher typical speeds at fixed temperature and are much more easily accelerated; i.e., they are much more mobile. As a result, it is the motion of the electrons, not the ions, that is responsible for the transport of heat and electricity through plasma [5].

The electron transport coefficients, the electrical conductivity, $\sigma$, and the thermal conductivity, $\kappa$, are evaluated using their basic definitions [6].

$$
\begin{gathered}
\sigma=\frac{e^{2} n_{e}}{m_{e} v_{e}} \\
\kappa=\frac{n_{e} k_{B}^{2} T_{e}}{m_{e} v_{e}}
\end{gathered}
$$

where $e$ and $m_{e}$ is the charge and mass of electron, respectively, $n_{e}$ is the electron density, $T_{e}$ is the electron temperature, $k_{B}$ is the Boltzmann constant and $v_{e}$ is the electron collision frequency. The collisions between electronneutral, electron-ion and electron-electron play an important role. Hence, we may assume that the overall electron collision frequency, $v_{e}$, is the sum of the electron-neutral collision frequency, electron-ion frequency and electronelectron collision frequency

$$
v_{e}=v_{e n}+v_{e i}+v_{e e}
$$

The plasma-neutral collision usually determines the kinetics of the motion. The electron-neutral collision frequency is given as [7]

$$
v_{e n}=n_{n}\left\langle\sigma_{e n} v_{t h}\right\rangle
$$

$n_{n}$ is the neutral atom density, $\sigma_{e n}$ the electron-neutral collision cross-section and $v_{t h}$ is the electron thermal velocity. In practical units, the electron-neutral collision frequency is given by [1]

$$
v_{e n}=2.60 \times 10^{4} \sigma_{e n}{ }^{2} n_{n} T_{e}^{1 / 2}
$$


( $T$ in $K, n_{n}$ in $m^{-3}$ and $\sigma_{e n}$ is the sum of the radii of the colliding particles).

The electron-ion collision frequency was described by the SpitzerHarm formula [8].

$$
v_{e i}=\frac{n_{e} e^{4} \ln \Lambda}{4 \pi \varepsilon_{0}^{2} m_{e}^{1 / 2}\left(e T_{e}\right)^{3 / 2}}
$$

where $\varepsilon_{0}$ is the permittivity of vacuum and $\ln \Lambda$ is the Coulomb logarithm given as:

$$
\ln \Lambda=23-\ln \left[\frac{n_{e}^{1 / 2} T_{e}^{-3 / 2}}{10^{2}}\right]
$$

In practical units, the electron-ion collision frequency is given by [1]

$$
\begin{aligned}
& v_{e i}=3.62 \times 10^{-6} n_{i} T_{e}^{-3 / 2} \ln \Lambda \\
& \Lambda=1.23 \times 10^{7} \frac{T_{e}^{3 / 2}}{n_{e}^{1 / 2}}
\end{aligned}
$$

Electron-electron collision frequency was based on the well known expression [9] $v_{e e} \approx \sqrt{2} v_{e i}$

\section{Experimental Set-up:}

The experimental set-up is designed to produce steady state lowpressure, direct current glow discharge plasma. Fig. (1) represents a schematic diagram of the experimental equipment and the electrical circuit. The discharge source is a Pyrex cylindrical tube with $20 \mathrm{~cm}$ inner diameter and $26 \mathrm{~cm}$ in length. A flange mounted two aluminum sheets were sealed at both ends of the tube. A stationary DC-glow discharge was generated between the electrode system which was made movable forward and backward inside the tube. It consists of two parallel copper electrodes with $7 \mathrm{~cm}$ diameter. The axial distance between the electrodes keeps fixed at $7 \mathrm{~cm}$ apart. The tube evacuation is carried out using a gas rotary pump to about $10^{-3}$ torr. Argon and helium are used here as plasma-forming gas during measurement to avoid possible negative effect of complex chemical reactions when a non-inert gas is applied [10]. The gas discharge was run from $2 \mathrm{kV}$ dc power supply with a rheostat ballast. The discharge current was varied between 2 and $20 \mathrm{~mA}$. 


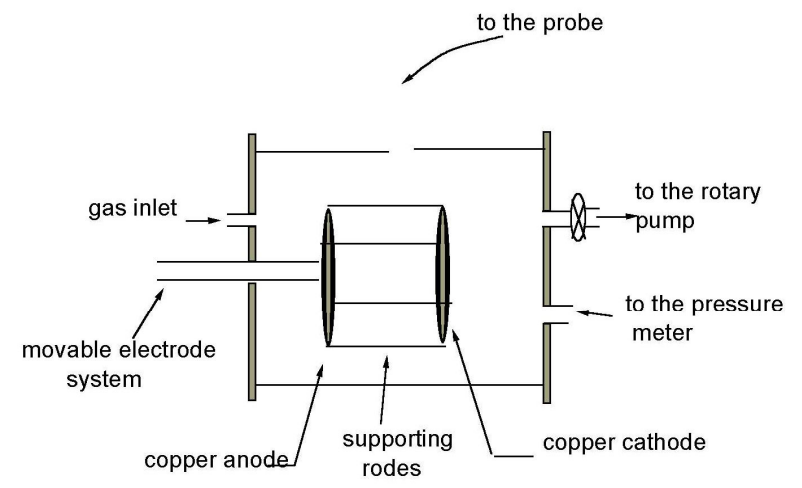

(a)

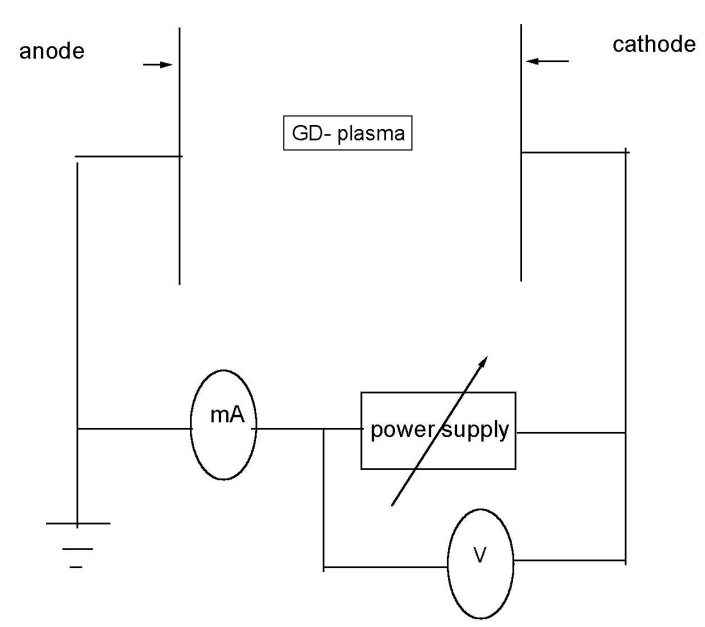

(b)

Fig. (1): A schematic diagram for (a) the experimental equipment and (b) The electric circuit.

In low-pressure glow discharge plasmas, Langmuir probes as a diagnostic tool are known for their ability to provide local measurements of very important plasma parameters, namely the electron density and electron temperature [11 \& 12]. The double probe used here consists of two identical cylindrical tungsten wire of $0.19 \mathrm{~mm}$ diameter and a length of $2 \mathrm{~mm}$. The separating distance between the two electrode centers was $2 \mathrm{~mm}$.

Measurements were made at a discharge voltage of $400 \mathrm{~V}$ for two different argon gas pressures: $\mathrm{p}=13.3$ and $\mathrm{p}=26.6 \mathrm{~Pa}$, and $750 \mathrm{~V}$ for two 
different helium gas pressures: $\mathrm{p}=53.2$ and $\mathrm{p}=79.8 \mathrm{~Pa}$, respectively. For each fixed bias point, the probe current and voltage were measured at each spatial (axial) location from the cathode surface to the anode in the discharge chamber. Fig. (2) shows a typical double-probe I-V characteristic curve. Maximum current through the double-probe circuit is determined by positive ion saturation currents $\left(i_{p 1,2}\right)$ to both probes. The electron temperature $T_{e}$ has been determined from a quite simple relation between the parameter $\Gamma$, defined as [13 \& 14],

$$
\Gamma=\frac{\sum\left|i_{p}\right|}{i_{e 2}}-1
$$

and the potential difference between probes $V$ :

$$
\ln \Gamma \sim-\frac{e}{k T_{e}} V
$$

That is, the reciprocal value of the slope of the semi-log plot $\ln \Gamma(V)$ versus $V$ equals the electron temperature in $\mathrm{eV}$.

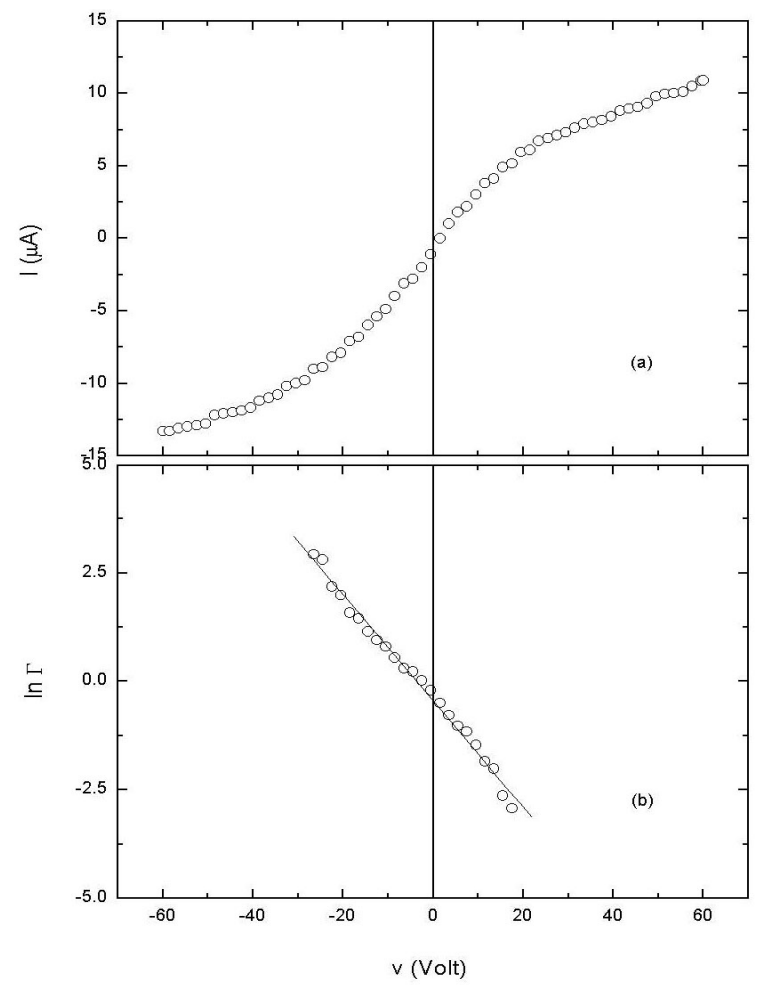

Fig. (2): (a) Typical double probe characteristic curve and (b) Electron temperature. 
The electron density $n_{e}=n_{i}$ has been determined from the ion saturation current, $I_{p}=I_{i s}$ of the double-probe characteristic curve using [15].

$$
I_{i s}=e n_{i} v_{i, t h} A
$$

where $A$ is the probe collecting area and $v_{i, t h}$ is the ion thermal speed given by $\left(2 k_{B} T_{e} / m_{i}\right)^{1 / 2}$, where $k_{B}$ is Boltzmann's constant, $T_{e}$ is the electron temperature and $m_{i}$ is the ion mass.

The neutral atom density, $n_{n}$, is computed from the gas pressure and temperature using ideal gas law $\left(n_{n}=P / k T_{g}\right)$. The neutral gas temperature, $T_{g}$, in glow discharges has been studied previously by means of Doppler broadening, thermal couple and via a manometer probe [16,17]. Recently, Rayleigh scattering of laser radiation is used to measure the neutral gas temperature [18]. In this work $T_{g}$ is $\sim 300 \mathrm{~K}$ as measured using a thermal couple.

\section{Results and Discussion:}

Regarding the low-pressure glow discharge plasma characterization, one is concerned with measuring the electron temperature, $T_{e}$, the electron density, $n_{e}$ and the neutral gas density $n_{n}$. To this purpose, a double Langmuir probe has been used, and the current-voltage (I-V) characteristic for argon and helium glow discharges, at different discharge voltage and gas pressure, along the tube axis from the cathode surface to the anode were measured. Values of the electron temperature are obtained by the logarithmic plot method according to Eq. (10). Values of $\Gamma$ can be obtained using the I-V characteristic curve, then a plot of $\ln \Gamma$ against $V$ yields a straight line, the slope of which gives the value of $T_{e}$, Eq. (11). The measured values of $T_{e}$ are about $(0.98-6.30 \mathrm{eV})$ at discharge voltage of 400 volt for two different argon pressures 13.3 and $26.6 \mathrm{~Pa}$, and (2.86-8.14 eV) for helium discharge at $750 \mathrm{~V}$ and 53.2 and $79.8 \mathrm{~Pa}$, respectively. The electron density, $n_{e}$, can also be obtained from the ion saturation current, $I_{p}=I_{i s}$ of the double-probe I-V characteristic curve, with the values of $T_{e}$ determined previously, according to Eq.(12). The electron density, over the pressure and voltage ranges employed above, are about $\left(1.19-7.65 \times 10^{9}\right.$ $\left.\mathrm{cm}^{-3}\right)$ and $\left(6.70-8.18 \times 10^{9} \mathrm{~cm}^{-3}\right)$ for argon and helium plasmas, respectively. Over the discharge voltage and pressure employed in this work, values of electron temperature, $T_{e}$, and electron density, $n_{e}$, agree reasonably well with other values given in the literature $[19,20]$. The neutral atom density, $n_{n}$, is computed from the gas pressure and temperature using ideal gas law $\left(n_{n}=P /\right.$ $k T_{g}$ ). The neutral gas temperature, $T_{g}$, measured using a thermal couple rises above room temperature by about $20 \mathrm{~K}$, and reaches a maximum of about 320 $\mathrm{K}$ at $2-4 \mathrm{~mm}$ from the cathode, under the voltage and pressure investigated, then it decreases slightly to room temperature along the tube axis to the anode. Hence, $T_{g}$, is taken to be $300 \mathrm{~K}$ approximately. 
Calculations of the electrical conductivity, $\sigma$, and thermal conductivity, $\kappa$, using Eqs. (1) and (2) necessitated calculations of the electron collision frequencies from Eqs. (5) to (9), respectively. With the experimental values of $T_{e}, n_{e}$ and $n_{n}$ which have been employed also to calculate $v_{e}$, the values of $\sigma$ and $\kappa$ were calculated.

The calculated electrical conductivity, $\sigma$, is plotted in Figs. 3(a) and 4(a) for argon and helium plasmas. Figs. 3(a) and 4(a) show the temperature dependence of $\sigma$, with pressure as parameter. Figure 3(a) clarifies the electrical conductivity of argon plasma as a function of electron temperature ranging from 1-6.3 eV at two different gas pressures (13.3 and 26.6 Pa). This plot shows that $\sigma$ rises in the region of low temperatures $\left(T_{e}<2.5 \mathrm{eV}\right)$. At higher electron temperatures $\left(T_{e}>3 \mathrm{eV}\right) \sigma$ tend to be constant. Quite similar behavior of $\sigma$ appear in Fig. 4(a) for helium plasma at neutral gas pressures of 53.2 and $79.8 \mathrm{~Pa}$, and for an electron temperature in the range of 3-8 eV. It can be seen that $\sigma$ show a rapid increase in the region of temperatures between $3-3.5 \mathrm{eV}$. In the higher temperature region (above $4 \mathrm{eV}$ ) one can notice only a constant variation with temperature. The results of the electrical conductivity of argon and helium plasmas are insensitive to the changes in the high energy electrons.

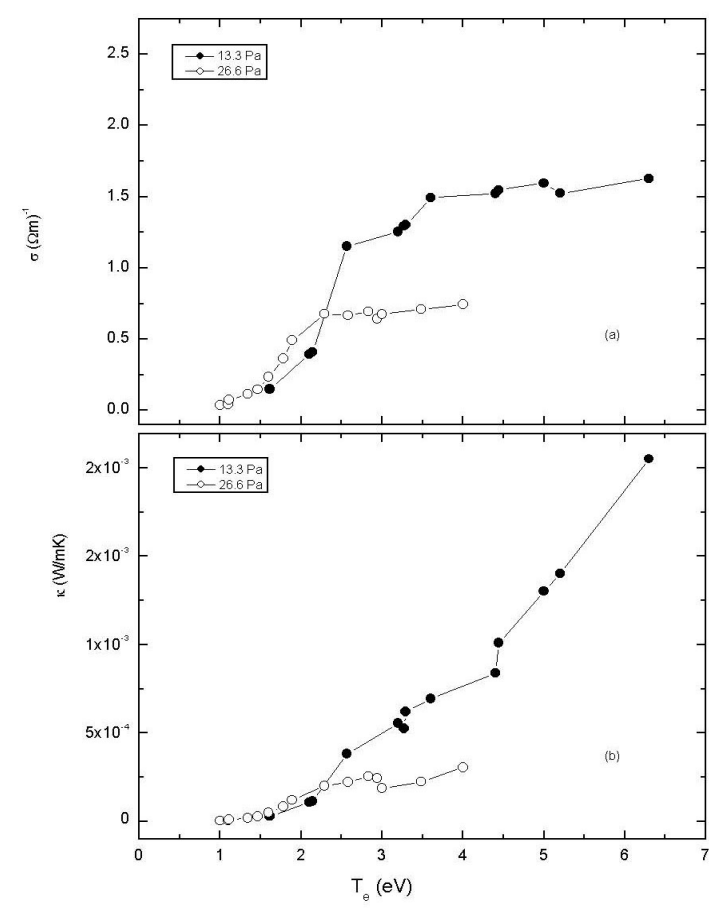

Fig. (3): (a) Electrical conductivity and (b) thermal conductivity in Ar Plasma. 
Figures 3(b) and 4(b) show the temperature dependence of the calculated thermal conductivity, $\kappa$. Different compared to the case of $\sigma$, the calculated values of $\kappa$ are virtually rises monotonically with the electron temperature. Generally, the electrical and thermal conductivities are seen to increase with temperature, which is an easily comprehensible feature ascribable mainly to the increasing thermal ionization [21].

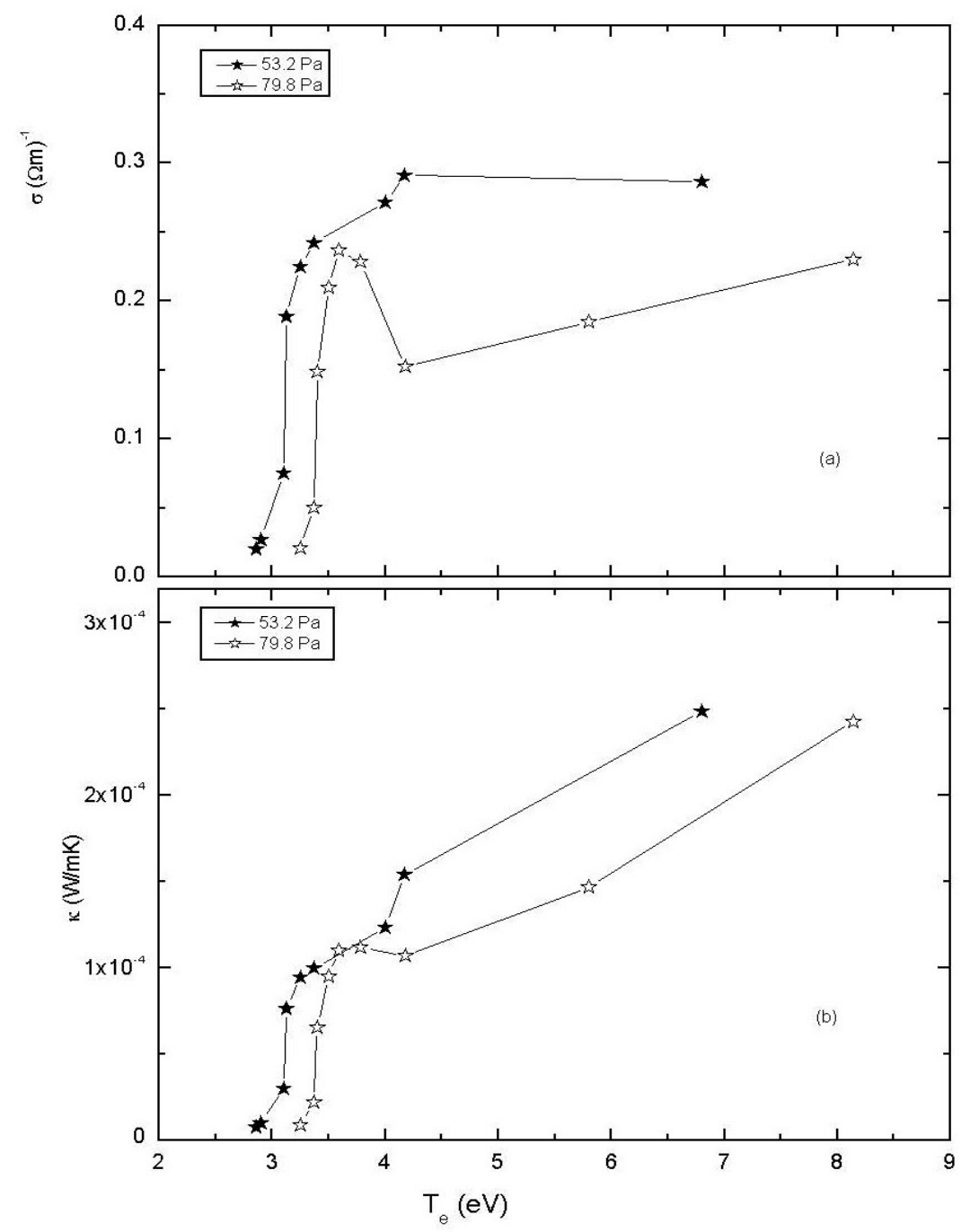

Fig. (4): (a) Electrical conductivity and (b) thermal conductivity in He Plasma. 
The pressure behavior of the electrical conductivity, $\sigma$, and thermal conductivity, $\kappa$, show interesting curves in Figs. 3 and 4 for various electron temperatures. It is clearly seen that, the calculated values of $\sigma$ and $\kappa$ are virtually independent of the pressure at lower electron temperatures (below 2.5 and $4 \mathrm{eV}$ for argon and helium plasmas), at that it decreases quite noticeable with the pressure above that temperatures. The inclusion of electron collision frequency may be significant in establishing these features shown in Figs. (3 \& 4) [4].

The behaviour of the electron collision frequency $v_{e}$ (inversely proportional to both $\sigma$ and $\kappa$ ) is characterized by a general increase with neutral gas pressure. This corresponds to an increasing number of collisions. However, proportionality is only observed for very small gas pressure (below $\sim 1 \mathrm{~Pa}$ ). Deviations from a direct proportionality become visible especially in the pressure regime over $20 \mathrm{~Pa}$ in case of low electron temperature $(2 \mathrm{eV})$. The general dependence of $v_{e}$ on the electron temperature can be explained by a higher average velocity with increasing temperature. For higher temperatures, collision events become more frequent, which results in an increase of $v_{e}$ [4].

An important character is given in Fig. (5) for argon discharge showing the temperature dependence of the ratio $\kappa / \sigma$ with the pressure as parameter. For helium discharge, the same values of $\kappa / \sigma$ was obtained (see Fig. (5)), although gas pressure range was higher than that of argon discharge. The calculated data in Fig. 5 show a good agreement with Wiedemann-Franz ratio, $\kappa / \sigma=\left(k^{2} / e^{2}\right) T$. On the other hand, Fig. (5) confirms that the ratio $\kappa / \sigma$ is independent on the gas pressure. Although, the general behavior of the present results of $\kappa / \sigma$ agrees with the expected theoretical law, they differ from that given by Novaković et al. [9].

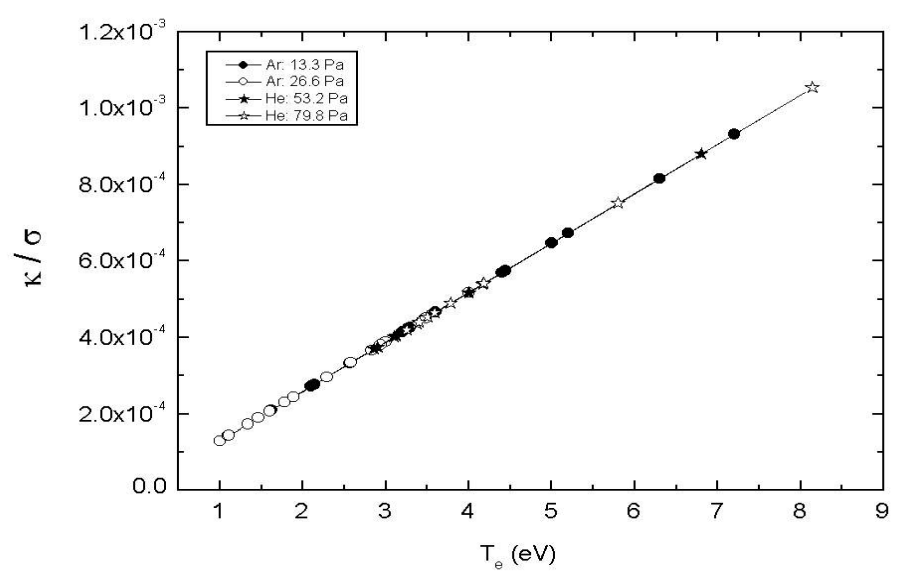

Fig. (5): Franz-Wiedemann ratio in Ar and He plasmas. 
Finally, a comparison of the electron collision frequencies, $v_{e}$, calculated via the experimental results of $T_{e}, n_{e}$ and $n_{n}$ are given in Table (1) for argon discharge and Table 2 for helium discharge, respectively. From the tables it is clearly seen that, electron-neutral collision frequencies, $v_{e n}$, over the whole range of electron temperatures, are so larger than the collision frequencies of electrons with the charged particles $v_{e i}$ and $v_{e e}$. So, the collisions with the ions in both argon and helium discharges are relatively insignificant in the whole electron temperature region considered here. As expected, of the three collision mechanisms studied here, the electron-neutral collisions play the main role in evaluating conductivity over other effects which include electron-ion and electron-electron, with the extra characterization of low-pressure weakly ionized glow discharge plasma.

Table (1): Electron temperature and collision frequencies in Ar plasma $(\mathrm{P}=13.3 \mathrm{~Pa})$.

\begin{tabular}{|c|c|c|c|c|}
\hline$\overline{\mathrm{T}_{\mathrm{e}}(\mathrm{eV})}$ & $v_{\text {en }}\left(x E 8 s^{-1}\right)$ & $v_{\mathrm{ei}}\left(\times \mathrm{E} 5 \mathrm{~s}^{-1}\right)$ & $v_{\mathrm{ee}}\left(\mathrm{xE5 \textrm {s } ^ { - 1 } )}\right.$ & $v_{\text {total }}\left(\mathrm{xE} \mathrm{E} \mathrm{\textrm {s } ^ { - 1 }}\right)$ \\
\hline 1.61 & 8.318 & 0.648 & 0.916 & 8.320 \\
\hline 1.62 & 10.29 & 0.785 & 1.110 & 10.298 \\
\hline 2.10 & 10.49 & 1.423 & 2.012 & 10.49 \\
\hline 2.14 & 12.34 & 1.683 & 2.380 & 12.35 \\
\hline 2.56 & 5.855 & 1.730 & 2.446 & 5.859 \\
\hline 3.20 & 5.837 & 1.475 & 2.086 & 5.840 \\
\hline 3.27 & 6.666 & 1.513 & 2.139 & 6.670 \\
\hline 3.29 & 6.729 & 1.764 & 2.495 & 6.733 \\
\hline 3.60 & 7.366 & 1.738 & 2.457 & 7.370 \\
\hline 4.40 & 8.229 & 1.453 & 2.054 & 8.232 \\
\hline 4.44 & 8.344 & 1.720 & 2.432 & 8.348 \\
\hline 5.00 & 8.728 & 1.740 & 2.461 & 8.732 \\
\hline 5.20 & 9.693 & 1.882 & 2.662 & 9.697 \\
\hline 6.30 & 9.649 & 1.729 & 2.445 & 9.653 \\
\hline
\end{tabular}

Table (2): Electron temperature and collision frequencies in He plasma

\begin{tabular}{|c|c|c|c|c|}
\hline $\mathrm{T}_{\mathrm{e}}(\mathrm{eV})$ & $v_{\mathrm{en}}\left(\mathrm{x} \mathrm{E} 8 \mathrm{~s}^{-1}\right)$ & $v_{\mathrm{ei}}\left(\mathrm{xE} 4 \mathrm{~s}^{-1}\right)$ & $v_{\mathrm{ee}}\left(\mathrm{x} \mathrm{E} 4 \mathrm{~s}^{-1}\right)$ & $v_{\text {total }}\left(x \mathrm{E} 8 \mathrm{~s}^{-1}\right)$ \\
\hline 3.25 & 9.405 & 0.434 & 0.613 & 9.405 \\
\hline 3.37 & 8.606 & 0.871 & 1.226 & 8.606 \\
\hline 3.40 & 8.444 & 2.3734 & 3.356 & 8.445 \\
\hline 3.50 & 8.482 & 3.184 & 4.503 & 8.483 \\
\hline 3.59 & 8.293 & 3.374 & 4.770 & 8.294 \\
\hline 3.78 & 8.716 & 3.189 & 4.509 & 8.717 \\
\hline 4.18 & 1.312 & 2.790 & 3.946 & 13.12 \\
\hline 5.80 & 1.108 & 1.820 & 2.574 & 11.08 \\
\hline 8.14 & 8.943 & 1.147 & 1.623 & 8.944 \\
\hline
\end{tabular}




\section{Conclusions:}

1. We measured the double probe I-V characteristic curve in both argon and helium discharges. From these curves we inferred values of the electron temperature and density. In our case, the electron temperature and density for argon discharge is lower than for helium discharge. This may be due to the higher ionization potential of helium atom compared with its value for argon atom.

2. High energy of the electrons (electron temperature, $T_{e}$ ) implies high plasma conductivity. The thermal conductivity, $\kappa$, is virtually rises monotonically with electron temperature.

3. The electrical conductivity, $\sigma$, is dominated by low energy electrons and insensitive to the changes in the high energy region.

4. Both electrical and thermal conductivity is independent of the pressure at lower electron temperature, and decreases noticeably with the pressure at higher electron temperature.

5. Wiedemann-Franz ratio is direct proportional with electron temperature and independent on the gas pressure.

6. Electron-neutral collisions play the main role in evaluating conductivity over other collision effects, and offering the possibility to treat the glow discharge plasma as weakly ionized.

7. The most important result of this work is that, the simple expressions for $\sigma$ and $\kappa$, Eqs. (1)and (2), does not provide a sufficiently accurate value of the plasma conductivities, since they based on a scalar effective electron collision frequency, $v_{e}$, Eqs. (5) to (9). We believe that to obtain more accurate values of $\sigma$ and $\kappa$, a general form of the plasma's conductivity considering the effective electron collision frequency, $v_{e}$, represented by the more general form depending on the electron energy distribution and the energy dependence of the corresponding collision cross section, Eq. (4), must be used.

\section{References:}

1. J. A. Bittencourt, "Fundamentals of plasma physics", Pergamon Press, Oxford (1986).

2. D. P. Lymberopoulos and D. J. Economou, J. Res. Natl. Stand. Technol. 100, 473 (1995).

3. H. W. Drawin, "Collision and transport cross-sections", In "Plasma Diagnostics", Published by the American Institute of Physics, United State of America, 1995.

4. P. Scheubert, "Modeling and diagnostics of low pressure plasma discharges", http://tumb1.biblio.tu-muenchen.de/publ/diss/ei/2002/scheubert. 
5. Z. Donko, P. Hartmann and K. Kutasi, "On the reliability of low pressure Dcglow discharge modeling", XXVIIth ICPIG, Eindhoven, the Netherlands (2005).

6. M. Mitchner and C. H. Kruger, "Partially ionized gases", Wiley (1973).

7. Roy Subrata and B. P. Pandey, "Element based hydrodynamic sheath model", 33rd Plasma dynamics and lasers conference, Maui, Hawaii, (2002).

8. J. T. Gudmundsson and M. A. Lieberman, Plasma Sources Sci. Technol. 6, 540 (1997).

9. N. V. Novaković, S. M. Stojiković and D. Z. Gajić, Facta Universitatis Series: Physics, Chemistry and Technology 3, 1 (2004).

10. Liu, C., Wang, J., Yu, K. I., Eliasson, B., Xia, Q., Xue, B. and Zhang, Y., J. Electrostatics 54, 149 (2002).

11. D. Fang and R. K. Marcus, Spectrochem. Acta B45, 1053 (1990).

12. A. Bogaerts, A. Quentmeier, N. Jakubowski and R. Gijbels, Spectrochim. Acta B50, 1337 (1995).

13. E. O. Johnson and L. Malter, Phys. Rev. 80, 58 (1950).

14. V. Margetić and D. Veža, Fizika A7 (2), 49 (1998).

15. C. Thompson, A.Barkan, N. D`Angelo and R. L. Merlino, Phys. Plasmas 4, 2331 (1997).

16. S. K. Ohorodnik and W. W. Harrison, J. Anal. Atom. Spectrom. 9, 991 (1994).

17. N. I. Uzelac and F. Leis, Spectrochim. Acta B47, 877 (1992).

18. G. Gamez, A. Bogaerts, F. Andrade and G. M. Hieftje, Spectrochim. Acta B59, 435 (2004).

19. E. Passoth, P. Kudrna, C. Csambal, M. Tichy and V. Helbig, J. Phys. D: Appl. Phys. 30, 1763 (1997).

20. M. Belkin, J. A. Caruso, S. J. Cristopher and R. K. Marcus, Spectrochim. Acta B53, 1197 (1998).

21. R. N. V. Novaković, B. C. Milić, S. M. Stojiković and D. Z. Gajić, Facta Universitatis Series: Physics, chemistry and technology 2, 285 (2003). 